\title{
Anomalía de Ebstein y preeclampsia, asociación infrecuente: reporte de caso y revisión de la literatura
}

\section{Ebstein's anomaly and preeclampsia, a rare association: case report and literature review}

\section{Jesús A. Velásquez-Penagos ${ }^{1,2,6 *}$, Ana T. Ospina-Pérez², Sarita Oyuela-Gómez ${ }^{3}$, Edison Muñoz-Ortiz ${ }^{4,5,6}$, Jairo A. Gándara-Ricardo ${ }^{4,5,6}$ y Juan P. Flórez-Muñoz $4,5,6$}

${ }^{1}$ Departamento de Ginecología y Obstetricia, Hospital Universitario San Vicente Fundación, Medellín; ${ }^{2}$ Departamento de Ginecología y Obstetricia, Facultad de Medicina, Universidad de Antioquia, Medellín; ${ }^{3}$ Facultad de Medicina, Universidad de Antioquia, Medellín; ${ }^{4}$ Departamento de Cardiología, Hospital Universitario San Vicente Fundación, Medellín; ${ }^{5}$ Departamento de Cardiología, Facultad de Medicina, Universidad de Antioquia, Medellín; ${ }^{6}$ Clínica Cardiobstétrica, Hospital Universitario San Vicente Fundación, Medellín. Colombia

\section{Resumen}

Se presenta el caso de una gestante con cardiopatía congénita no estudiada que acudió al servicio de urgencias en la semana 25 + 4 por palpitaciones, con evidencia de hipertensión arterial desde el ingreso. Los estudios diagnósticos revelaron anomalía de Ebstein, con gran compromiso de cavidades derechas y asociado a comunicación interauricular. También se documentó preeclampsia lejos del término, con restricción grave del crecimiento intrauterino. Durante la estancia hospitalaria, y ante negativa de la paciente a finalizar la gestación, desarrolló síntomas de congestión pulmonar por sobrecarga. Una vez falleció el feto in utero y después de inducir el parto, remitieron los síntomas cardiovasculares y se controló la hipertensión.

Palabras clave: Anomalía de Ebstein. Preeclampsia. Cardiopatía en embarazo.

\section{Abstract}

We present the case of a pregnant woman with previously undiagnosed congenital heart disease, who presented to the emergency department at week $25+4$ due to palpitations, with evidence of arterial hypertension from admission. Diagnostic studies revealed Ebstein's anomaly, with great involvement of the right cavities and associated with atrial septal defect. Preeclampsia was also documented far from term, associated with severe intrauterine growth restriction. During the hospital stay and due to the refusal of the patient to end the pregnancy, she developed symptoms of pulmonary congestion due to overload, once the fetus died in utero and after inducing labor, the cardiovascular symptoms remitted and hypertension was controlled.

Keywords: Ebstein's anomaly. Preeclampsia. Heart disease in pregnancy.

\section{Introducción}

La anomalía de Ebstein (AE) explica el 0,5\% de las enfermedades congénitas cardiacas. Consiste en el desplazamiento apical de los velos de la válvula tricúspide por una alteración en la delaminación de las valvas, causando insuficiencia valvular significativa, disminución del ventrículo derecho funcional e
Correspondencia:

*Jesús Velásquez

Fecha de recepción: 15-03-2021

Fecha de aceptación: 04-11-2021

E-mail: jesus.velasquez@sanvicentefundacion.com DOI: 10.24875/RECHOG.M21000040

0048-766X / ๑ 2021 Sociedad Chilena de Obstetricia y Ginecología. Publicado por Permanyer. Éste es un artículo open access bajo la licencia CC BY-NC-ND (https://creativecommons.org/licenses/by-nc-nd/4.0/).
Disponible en internet: 07-02-2022 Rev Chil Obstet Ginecol. 2021;86(6):529-537

www. rechog.com 
insuficiencia cardiaca derecha, asociándose a preexcitación y cortocircuitos a nivel auricular, lo que puede originar embolias paradójicas e hipoxemia'. Los cambios fisiológicos durante el embarazo requieren la adaptación del sistema cardiovascular, por lo que exponen a la mujer con enfermedad cardiovascular preexistente a riesgos importantes, tales como falla cardiaca, arritmias letales, pérdida precoz del embarazo, parto pretérmino, muerte fetal e incluso la muerte materna $^{2}$. La enfermedad cardiovascular, tanto congénita como adquirida, es la primera causa de mortalidad materna en los países desarrollados ${ }^{3}$, y los trastornos hipertensivos de la gestación son la primera causa de mortalidad materna global ${ }^{4}$. Por ello, la aparición de preeclampsia (PE) en gestantes con cardiopatía congénita puede ensombrecer su pronóstico ${ }^{5}$.

Presentamos el caso de una paciente con AE, complicada con $\mathrm{PE}$ lejos del término, y la revisión narrativa de la literatura que permite, por un lado, describir las complicaciones más frecuentes de la $A E$ durante la gestación, y por otro lado la búsqueda de casos similares para intentar establecer si existe algún tipo de asociación (causal, fisiológica o temporal) entre la $\mathrm{AE}$ y la $\mathrm{PE}$.

\section{Presentación del caso}

Paciente atendida en un hospital de referencia de alta complejidad que cuenta con una clínica cardioobstétrica desde el año 2015 (programa de atención multidisciplinario para atención de gestantes con cardiopatía), conformado por cardiología, ginecoobstetricia, anestesia, enfermería y todas las subespecialidades de apoyo, tanto de cardiología como de ginecoobstetricia y neonatología, siendo un grupo profesional estable desde su inicio y que lleva un Registro de Embarazo y Enfermedad Cardiaca (REMEC) desde entonces (actualmente 550 pacientes).

Primigrávida de 36 años, O positivo, que inició sus controles prenatales en la semana 14 en otra institución, de carácter ambulatorio y de baja complejidad de atención, refiriendo antecedente de enfermedad cardiaca, la cual no había sido clasificada. Se le solicitó ecocardiografía transtorácica, la cual fue realizada en la semana 17 de gestación, en otra institución, cuya conclusión fue la siguiente: «Cavidades izquierdas normales, compromiso de cavidades derechas, anomalía de Ebstein, insuficiencia mitral y tricuspídea moderada. Válvulas aórtica y pulmonar normales, sin trombos intracavitarios, tabiques interauricular e interventricular normales. Fracción de expulsión del ventrículo izquierdo (FEVI): 50-55\%". Fue evaluada ambulatoriamente por un cardiólogo y un ginecoobstetra (de manera separada y en otra institución), y no se ordenó ningún manejo especifico. Consultó en la semana $25+4$ en el servicio de urgencias generales de una institución de segundo nivel de atención, por palpitaciones, que iniciaron en la gestación y que se fueron incrementando en frecuencia y se tornaron muy sintomáticas, hasta impedir la realización de sus labores cotidianas. Allí además encuentran hipertensión y altura uterina baja para la edad gestacional. Aplicaron $12 \mathrm{mg}$ de betametasona por vía intramuscular como inicio de maduración pulmonar fetal, y se decide el traslado a nuestra institución para manejo integral en la clínica cardioobstétrica.

La paciente no tenía otros antecedentes médicos relevantes, y no fumaba ni tomaba licor.

En la exploración física de ingreso se encontró presión arterial $151 / 90 \mathrm{mmHg}$, pulso $74 / \mathrm{min}$, frecuencia respiratoria 16/min, saturación de oxígeno 94\% (al aire ambiente), fetocardia 136/min y reflejos osteotendinosos $++/+++$.

En el examen cardiovascular no había ingurgitación yugular y se auscultó un soplo sistólico de grado II/VI de predominio en focos de la punta (mitral y tricuspídeo). No se auscultaron ruidos pulmonares patológicos. No presentaba edemas. La altura uterina era de $21 \mathrm{~cm}$.

La paciente no presentaba síntomas de irritación cortical (cefalea, fotopsias, tinnitus) ni dolor en el hipocondrio derecho o el epigastrio.

La clasificación funcional cardiovascular de la New York Heart Association correspondía a grado I.

Fue hospitalizada en la unidad de cuidados especiales maternos para tratamiento integral en la clínica cardioobstétrica.

La ecografía gestacional realizada reportó feto de 25 + 4 semanas, podálico, placenta fúndica anterior, índice de líquido amniótico disminuido, anatomía fetal normal (incluso el corazón) y peso fetal aproximado de $439 \mathrm{~g}$, creciendo por debajo del percentil 3. El Doppler de arteria uterina fue reportado como «marcadamente patológico, sospecha de insuficiencia placentaria. Restricción del crecimiento intrauterino (RCIU) grave».

El monitoreo Holter se reportó como «frecuencia cardiaca promedio de 78 latidos por minuto, con latidos auriculares prematuros ocasionales con un episodio de taquicardia atrial no sostenida, periodos de ritmo auricular ectópico de adecuada frecuencia. Aumento de automatismo auricular».

Se decidió realizar ecocardiografía transesofágica, dado que en la transtorácica, cuando se realizó el test 


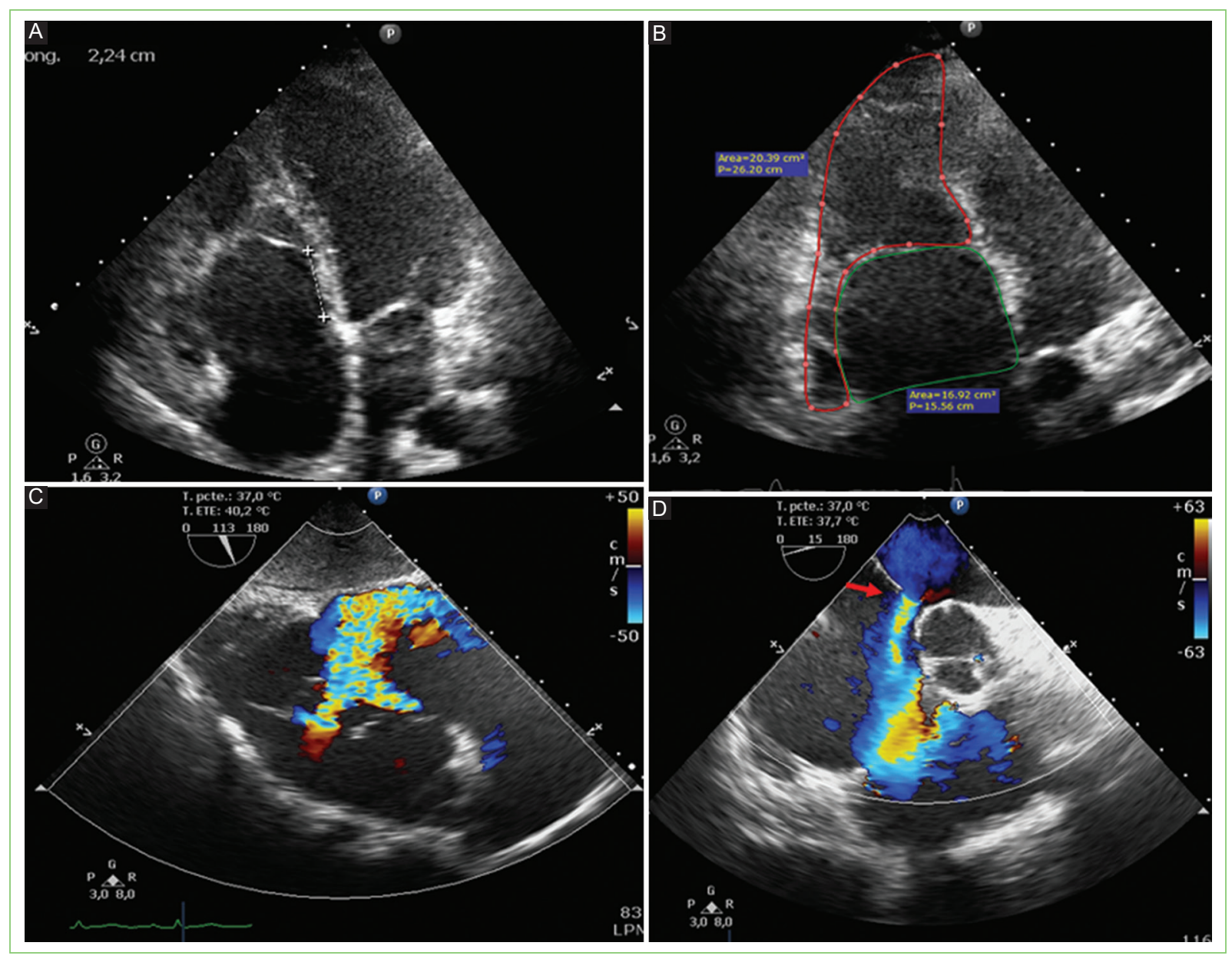

Figura 1. A: ecocardiograma transtorácico en ventana de cuatro cámaras que muestra desplazamiento apical de la inserción del velo septal, a $22 \mathrm{~mm}$ del anillo, diagnóstico de anomalía de Ebstein. B: ventana de cuatro 4 cámaras, zoom de ventrículo derecho, en rojo ventrículo derecho funcional y en verde ventrículo derecho atrializado.

C: ecocardiograma transesofágico en ventana transgástrica, Doppler color que muestra insuficiencia tricúspide grave excéntrica con dos chorros. D: ecocardiograma transesofágico, la flecha roja señala una comunicación interauricular tipo ostium secundum, y el Doppler color revela flujo sistólico de izquierda a derecha.

de solución salina, se observó un gran paso de burbujas a las cavidades izquierdas. Se reportó «enfermedad de Ebstein, comunicación interauricular tipo ostium secundum, insuficiencia tricuspídea grave, ventrículo derecho muy dilatado con función sistólica preservada (FEVI 62\%), ventrículo izquierdo normal sin evidencia de hipertensión pulmonar» (Fig. 1).

Con los hallazgos ecocardiográficos y clínicos, se clasificó el riesgo de presentar desenlaces cardiovasculares maternos adversos (arritmia que requiriera intervención, edema pulmonar, accidente cerebrovascular, parada cardiaca e incluso la muerte), según la clasificación de riesgo en gestantes con cardiopatía de la Organización Mundial de la Salud modificada ${ }^{6}$, como II-III (por la insuficiencia valvular grave y la comunicación interauricular), que supone un riesgo del 10-19\%. Según la escala de riesgo cardiovascular en el embarazo CARPREG (Cardiac Disease in Pregnancy Study), le correspondieron 2 puntos (10\% de riesgo) 7 .

El reporte de la proteinuria en 24 horas fue de $506 \mathrm{mg}$, lo que confirmó el diagnóstico de PE, hasta ese momento sin criterios clínicos ni de laboratorio de gravedad.

Dados los riesgos de por sí elevados de desenlaces adversos de ambas condiciones por separado (AE y $\mathrm{PE})$, que no existen escalas de riesgo que combinen estos parámetros y que, además, cursaba con RCIU grave y estaba por debajo del límite de viabilidad fetal (26 semanas o $700 \mathrm{~g}$ de peso fetal), se decidió, por parte del grupo de la clínica cardioobstétrica, sugerir a 


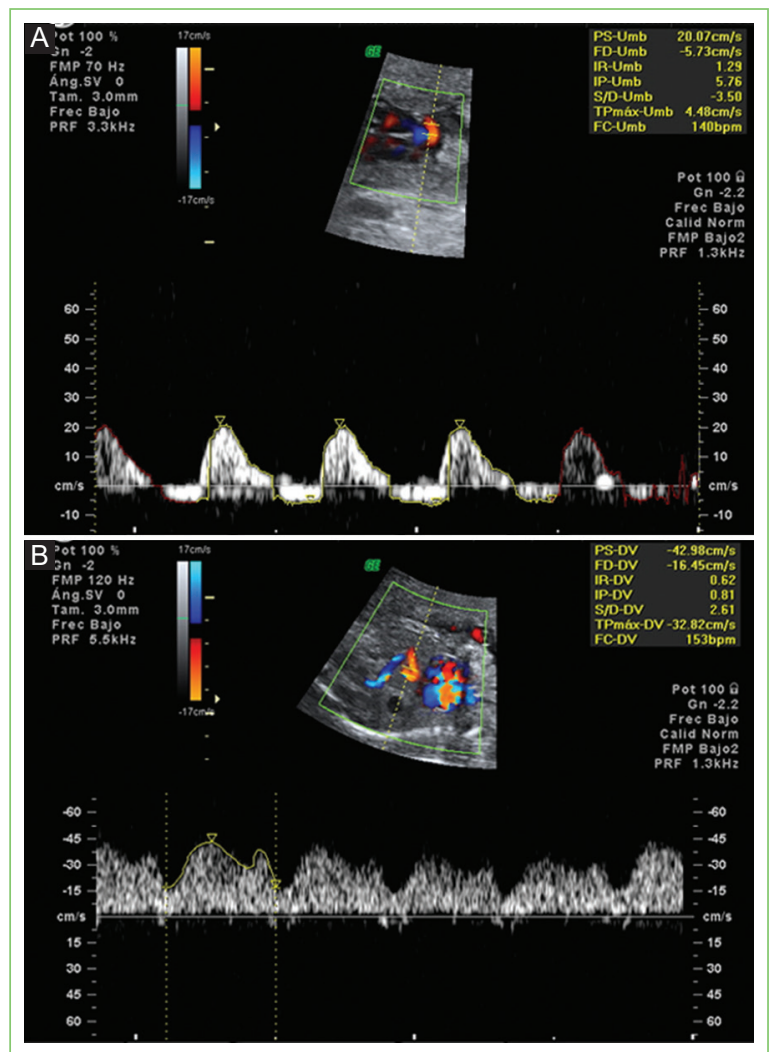

Figura 2. Doppler fetal realizado el día 7 de hospitalización. A: flujo reverso en la arteria umbilical. B: onda de ductus venoso fetal.

la paciente terminar la gestación. Se le explicó ampliamente la situación, pero decidió continuar con el embarazo. Por el incremento del automatismo auricular y los síntomas, tenía indicación de iniciar betabloqueador, el cual no se inició dado el RCIU grave y las expectativas expresadas por la paciente.

Se contó con el apoyo permanente de psicología y psiquiatría de enlace institucionales, quienes además de la familia brindaban apoyo emocional y acompañamiento del duelo. La paciente siempre manifestó ideas esperanzadoras frente a su condición.

Las cifras de presión arterial se mantuvieron en 140150/90-95 mmHg, sin requerimiento de antihipertensivo. Se administró enoxaparina, $40 \mathrm{mg} / \mathrm{día}$, como profilaxis tromboembólica.

Una semana después, por solicitud de la paciente, se realizaron pruebas de bienestar fetal que reportaron: «Doppler fetal con flujo diastólico reverso en arteria umbilical, con ductus normal, perfil biofísico 8/8, RCIU tipo III» (Fig. 2). Se explicó a la paciente el compromiso fetal, pero manifestó nuevamente su deseo de continuar con la gestación y esperar la evolución.

En el día 9 de hospitalización inicia disnea e hipoxemias moderadas (frecuencia respiratoria $24 / \mathrm{min}$, saturación de oxígeno 90-92\%), que mejoraron con oxígeno suplementario a bajo flujo (fracción inspirada de oxígeno al $32 \%$, con cánula nasal), y se interpretaron hallazgos por flujo sanguíneo de izquierda a derecha de la comunicación interauricular (CIA). Los gases arteriales evidenciaron una relación de presión arterial de oxígeno/fracción inspirada de oxígeno de 271 (hipoxemia moderada). Las cifras de presión arterial permanecían controladas sin antihipertensivos ni signos de irritación cortical. Se inició tratamiento diurético con furosemida, $20 \mathrm{mg} /$ día por vía intravenosa. En el día 15 hubo necesidad de incrementar la fracción inspirada de oxígeno al $50 \%$ por hipoxemia materna. Nuevamente, por solicitud de la paciente, se realizó ecografía gestacional para cálculo de peso fetal y nuevo Doppler, los cuales reportaron «peso de $553 \mathrm{~g}$ (crecimiento fetal por debajo del percentil 3), índice de líquido amniótico normal. Doppler fetal: flujo reverso en arteria umbilical, ductus venoso con onda A positiva». Se tomó determinó BNP materno, el cual fue de $16.8 \mathrm{pg} / \mathrm{ml}$ (normal), por lo que se interpretó que en el deterioro de la paciente estaba predominando la preeclampsia sobreimpuesta a una condición congénita cardiaca. Se realizó nuevamente reunión del grupo multidisciplinario y la decisión fue la misma, terminar gestación, pero la paciente persistía en su deseo de continuarla.

En el día 17 de hospitalización no se auscultó fetocardia y se confirmó la muerte fetal por ecografía, por lo que se inició la inducción del parto con misoprostol. Tras 24 horas se atendió el parto y se obtuvo un recién nacido muerto de $550 \mathrm{~g}$ de peso. La paciente permaneció 5 días más hospitalizada, toleró el desmonte de oxígeno y egresó en buenas condiciones. No aceptó método de anticoncepción al egreso. Se le explicó la necesidad de resolver primero su condición cardiaca antes de un nuevo embarazo y realizar una evaluación preconcepcional por parte del grupo multidisciplinario.

En la tabla 1 se presenta el resumen de los resultados de los exámenes de laboratorio, los hallazgos del Doppler fetal y las intervenciones realizadas a la paciente.

\section{Resultados}

Se llevó a cabo una búsqueda de la literatura en las bases de datos PubMed y EMBASE, en octubre de 2020, con los términos "Ebstein anomaly", "pregnancy", "preeclampsia" y "fetal growth restriction". La búsqueda no tuvo 
Tabla 1. Resumen de los resultados de laboratorio y las intervenciones realizadas

\begin{tabular}{|c|c|c|c|c|c|c|c|}
\hline Exámenes durante la hospitalización & Día 1 & Día 2 & Día 3 & Día 7 & Día 9 & Día 15 & Día 18 \\
\hline Hemoglobina (g/dl) & 11,9 & & 13,2 & 13,2 & & & 12,1 \\
\hline Plaquetas $/ \mathrm{mm}^{3}$ & 224 & 257 & 269 & 269 & 187 & & 287 \\
\hline Deshidrogenasa láctica (UI/I) & 322 & & 280 & 280 & 245 & & \\
\hline AST (U/I) & 30 & & 28 & 22 & 19 & 19 & \\
\hline ALT (U/I) & 32 & & 20 & 25 & 22 & 21 & \\
\hline Creatinina (mg/dl) & 0,79 & 0,75 & 0,7 & 0,7 & & 0,8 & \\
\hline Proteínas en orina 24 horas $(\mathrm{mg})$ & & 506,8 & & & & & \\
\hline BNP $(\mathrm{pg} / \mathrm{ml})$ & & & & & & 16,8 & \\
\hline $\begin{array}{l}\text { Hallazgos ecografía gestacional y Doppler fetal } \\
\text { Peso fetal estimado } \\
\text { Flujo arteria umbilical } \\
\text { Onda A ductus venoso }\end{array}$ & $493 \mathrm{~g}$ & & & $\begin{array}{l}\text { Reverso } \\
\text { Normal }\end{array}$ & & $\begin{array}{c}553 \mathrm{~g} \\
\text { Reverso } \\
\text { Normal }\end{array}$ & \\
\hline $\begin{array}{l}\text { Tratamiento materno } \\
\text { Betametasona } 12 \mathrm{mg} \text { intramuscular (una sola dosis) } \\
\text { Furosemida } 20 \mathrm{mg} / \text { día intramuscular } \\
\text { Enoxaparina } 40 \mathrm{mg} \\
\text { Nifedipino } 30 \mathrm{mg} \\
\text { Parto }\end{array}$ & $\begin{array}{l}x \\
x\end{array}$ & $x$ & $x$ & $\begin{array}{l}X \\
X\end{array}$ & $\begin{array}{l}x \\
x\end{array}$ & $\begin{array}{l}X \\
X \\
X\end{array}$ & $x$ \\
\hline
\end{tabular}

restricción de año, pero se limitó a artículos publicados en inglés y español que incluyeran casos en los que fuera la madre quien presentara $\mathrm{AE}$; se incluyeron estudios de cohorte y reportes de casos, y se excluyeron cartas al editor y revisiones narrativas de la literatura. Tres de los autores hicieron una selección de los artículos tras la lectura del resumen. Los estudios en otros idiomas o sin acceso al texto completo fueron excluidos.

De cada artículo se tomaron los siguientes datos: año, tipo de estudio, características maternas, complicaciones cardiovasculares, complicaciones obstétricas y resultados neonatales.

Después de eliminar los duplicados, quedaron 174 referencias. Se excluyeron 92 artículos porque era el feto quién presentaba $A E, 5$ de malformaciones cardiovasculares diferentes de la $A E, 17$ que no estaban en gestación, 6 revisiones de la literatura y 15 no relacionados con el tema, 8 artículos que estaban en otro idioma y 5 que no tenían disponible el texto completo. Finalmente, se revisaron 26 artículos.

De los artículos seleccionados, el primero se publicó en 1970 y el último en 2019. Se analizaron 229 pacientes y 374 embarazos (Tabla 2).

El promedio de edad de las gestantes en los reportes de caso fue de 26 (19-37) años, y el promedio de la edad gestacional fue de 32 (14-39) semanas. No se describió ningún caso de muerte materna asociado a $\mathrm{AE}$.

El defecto cardiovascular asociado con más frecuencia fue la CIA, en 42 (18.3\%) pacientes. Los síndromes de preexcitación se reportaron en 31 pacientes $(13,5 \%)$ y hubo $18(4,8 \%)$ casos de falla cardiaca ${ }^{8-16}$, aunque en uno de los artículos este desenlace se reportó como $<10 \%{ }^{17}$. La cianosis se reportó en 10 pacientes $(4.3 \%)^{10,11,18-20}$ y el bloqueo de rama derecha en $10(4.3 \%)^{12,14,19,21-24}$. Además, 27 (11.7\%) pacientes presentaron otro tipo de arritmia diferente del síndrome de preexcitación, y requirieron manejo farmacológico o eléctrico. Se reportaron cuatro casos de hipertensión pulmonar ${ }^{8,9,21}$ y cuatro casos de accidentes cerebrovasculares $^{12,13,16,25}$, tres de las pacientes en el posparto tardío, una de ellas presentó preeclampsia, y dos de estas cuatro pacientes eran portadoras de válvula protésica mecánica tricuspídea (en una por suspensión de anticoagulación y en otra por anticoagulación insuficiente con heparina de bajo peso molecular). Se reportó un caso de síndrome de Eissenmenger ${ }^{26}$. La mayoría de los partos fueron vaginales, en 137 (36,6\%) pacientes; hubo $72(19,25 \%)$ cesáreas y 10 partos instrumentados $(2,6 \%)$. El parto pretérmino se presentó en 68 embarazos (18,8\%). De los estudios que reportan el 
Tabla 2. Resumen de las características de los desenlaces cardiovasculares y obstétricos en gestantes con anomalía de Ebstein en los artículos revisados

\begin{tabular}{|c|c|c|c|c|c|c|c|c|c|c|c|}
\hline Autores & Año & Tіро & $\begin{array}{l}\text { Número } \\
\text { de } \\
\text { casos }\end{array}$ & $\begin{array}{l}\text { Número } \\
\text { de } \\
\text { embarazos }\end{array}$ & $\begin{array}{l}\text { Edad, } \\
\text { años* }\end{array}$ & $\begin{array}{c}\text { EG, } \\
\text { semanas* }\end{array}$ & $\mathrm{CIA}^{\dagger}$ & Cianosis $^{\dagger}$ & SPE' & Tipo de parto & $P^{\dagger}$ \\
\hline Littler $^{25}$ & 1970 & $\begin{array}{l}\text { Reporte } \\
\text { de } \\
\text { caso }\end{array}$ & 1 & 1 & 23 & 36 & 1 & No & No & Vaginal & 1 \\
\hline Schatz $^{38}$ & 1974 & $\begin{array}{l}\text { Reporte } \\
\text { de } \\
\text { caso }\end{array}$ & 1 & 1 & 21 & NR & No & No & No & Vaginal & 1 \\
\hline $\begin{array}{l}\text { Waickman } \\
\text { et al. }{ }^{19}\end{array}$ & 1984 & $\begin{array}{l}\text { Reporte } \\
\text { de } \\
\text { caso }\end{array}$ & 1 & 1 & 19 & 14 & No & 1 & No & Cesárea & No \\
\hline $\begin{array}{l}\text { Donnelly } \\
\text { et al. }^{10}\end{array}$ & 1991 & Cohorte & 12 & 42 & NR & NR & No & 2 & 2 & $\begin{array}{l}\text { Vaginal } 26 \\
\text { Cesárea } 3 \\
\text { Instrumentado } 3\end{array}$ & 4 \\
\hline $\begin{array}{l}\text { Connolly y } \\
\text { Warnes }^{28}\end{array}$ & 1994 & Cohorte & 42 & 111 & 25 & NR & 20 & No & 5 & $\begin{array}{l}\text { Vaginal } 28 \\
\text { Cesárea } 9\end{array}$ & 24 \\
\hline $\begin{array}{l}\text { Groves y } \\
\text { Groves }^{23}\end{array}$ & 1994 & $\begin{array}{l}\text { Reporte } \\
\text { de } \\
\text { caso }\end{array}$ & 1 & 1 & 24 & 37 & No & No & No & Instrumentado & No \\
\hline Misa y $\operatorname{Pan}^{8}$ & 2007 & $\begin{array}{l}\text { Reporte } \\
\text { de } \\
\text { caso }\end{array}$ & 1 & 1 & 25 & 34 & No & No & 1 & Instrumentado & No \\
\hline $\begin{array}{l}\text { Íñigo } \\
\text { et al. }{ }^{18}\end{array}$ & 2008 & $\begin{array}{l}\text { Serie } \\
\text { de } \\
\text { casos }\end{array}$ & 5 & 5 & NR & 36 & 2 & 2 & No & NR & 5 \\
\hline $\begin{array}{l}\text { Chopra } \\
\text { et al. }{ }^{28}\end{array}$ & 2010 & $\begin{array}{l}\text { Serie } \\
\text { de } \\
\text { casos }\end{array}$ & 4 & 6 & 25 & 19 & No & No & No & Instrumentado & 3 \\
\hline Suárez ${ }^{39}$ & 2011 & $\begin{array}{l}\text { Reporte } \\
\text { de } \\
\text { caso }\end{array}$ & 1 & 1 & 37 & 32 & No & No & 1 & Cesárea & Sí \\
\hline $\begin{array}{l}\text { Castro } \\
\text { et al. }{ }^{21}\end{array}$ & 2011 & $\begin{array}{l}\text { Reporte } \\
\text { de } \\
\text { caso }\end{array}$ & 1 & 1 & 20 & 38 & No & No & No & Instrumentado & No \\
\hline $\begin{array}{l}\text { Sheikh } \\
\text { et al. }\end{array}$ & 2011 & $\begin{array}{l}\text { Reporte } \\
\text { de } \\
\text { caso }\end{array}$ & 1 & 1 & 28 & 24 & No & No & No & Cesárea & 1 \\
\hline Cornet $^{40}$ & 2011 & $\begin{array}{l}\text { Reporte } \\
\text { de } \\
\text { caso }\end{array}$ & 1 & 1 & NR & 34 & No & No & No & Instrumentado & 1 \\
\hline $\begin{array}{l}\text { Gouton } \\
\text { et al. } .^{20}\end{array}$ & 2011 & Cohorte & 22 & 47 & NR & NR & No & 4 & 7 & NR & No \\
\hline $\begin{array}{l}\text { Zhao } \\
\text { et al. }^{12}\end{array}$ & 2012 & $\begin{array}{l}\text { Serie } \\
\text { de } \\
\text { casos }\end{array}$ & 4 & 4 & 32 & 37 & No & No & No & Cesárea 4 & 1 \\
\hline $\begin{array}{l}\text { Katsuragi } \\
\text { et al. } .^{13}\end{array}$ & 2013 & Cohorte & 13 & 27 & 32 & 37 & 4 & No & 6 & $\begin{array}{l}\text { Vaginal } 17 \\
\text { Cesárea } 2\end{array}$ & 2 \\
\hline $\begin{array}{l}\text { Parizad } \\
\text { et al. }{ }^{9}\end{array}$ & 2014 & $\begin{array}{l}\text { Reporte } \\
\text { de } \\
\text { caso }\end{array}$ & 1 & 1 & 34 & 32 & 1 & No & 1 & NR & No \\
\hline
\end{tabular}


Tabla 2. Resumen de las características de los desenlaces cardiovasculares y obstétricos en gestantes con anomalía de Ebstein en los artículos revisados (continuación)

\begin{tabular}{|c|c|c|c|c|c|c|c|c|c|c|c|}
\hline Autores & Año & Тіро & $\begin{array}{c}\text { Número } \\
\text { de } \\
\text { casos }\end{array}$ & $\begin{array}{c}\text { Número } \\
\text { de } \\
\text { embarazos }\end{array}$ & $\begin{array}{l}\text { Edad, } \\
\text { años* }\end{array}$ & $\begin{array}{c}\text { EG, } \\
\text { semanas* }\end{array}$ & $\mathrm{CIA}^{\dagger}$ & Cianosis $^{\dagger}$ & SPE ${ }^{\dagger}$ & Tipo de parto & $\mathbf{P P}^{\dagger}$ \\
\hline $\begin{array}{l}\text { Ranjbar } \\
\text { et al. }{ }^{11}\end{array}$ & 2014 & $\begin{array}{l}\text { Reporte } \\
\text { de } \\
\text { caso }\end{array}$ & 1 & & 23 & 29 & 1 & 1 & No & NR & 1 \\
\hline $\begin{array}{l}\text { Patel y } \\
\text { Jamnadas }^{14}\end{array}$ & 2014 & $\begin{array}{l}\text { Reporte } \\
\text { de } \\
\text { caso }\end{array}$ & 1 & 1 & NR & 37 & No & No & No & Cesárea & No \\
\hline $\begin{array}{l}\text { Lesniak } \\
\text { et al. }{ }^{24}\end{array}$ & 2015 & $\begin{array}{l}\text { Serie } \\
\text { de } \\
\text { casos }\end{array}$ & 10 & 10 & 24 & NR & 5 & No & No & NR & No \\
\hline $\begin{array}{l}\text { Houser } \\
\text { et al. }^{30}\end{array}$ & 2015 & $\begin{array}{l}\text { Serie } \\
\text { de } \\
\text { casos }\end{array}$ & 3 & 3 & NR & NR & 3 & No & No & Vaginal 3 & 1 \\
\hline $\begin{array}{l}\text { Lima } \\
\text { et al. }{ }^{17}\end{array}$ & 2016 & Cohorte & 82 & 82 & 27 & NR & 5 & No & 8 & $\begin{array}{l}\text { Vaginal } 43 \\
\text { Cesárea } 39\end{array}$ & 16 \\
\hline $\begin{array}{l}\text { Rusdi } \\
\text { et al. } .^{15}\end{array}$ & 2016 & $\begin{array}{l}\text { Reporte } \\
\text { de } \\
\text { caso }\end{array}$ & 1 & 1 & 27 & 30 & No & No & No & Cesárea & 1 \\
\hline Sharma ${ }^{41}$ & 2018 & $\begin{array}{l}\text { Reporte } \\
\text { de } \\
\text { caso }\end{array}$ & 1 & 1 & 24 & 39 & No & No & No & cesárea & No \\
\hline $\begin{array}{l}\text { Kanoh } \\
\text { et al. }{ }^{16}\end{array}$ & 2018 & $\begin{array}{l}\text { Serie } \\
\text { de } \\
\text { casos }\end{array}$ & 17 & 23 & 31 & 38 & No & No & No & $\begin{array}{l}\text { Vaginal } 14 \\
\text { Cesárea } 9\end{array}$ & 5 \\
\hline Katamreddy ${ }^{27}$ & 2019 & $\begin{array}{l}\text { Reporte } \\
\text { de } \\
\text { caso }\end{array}$ & 1 & 1 & 19 & NR & No & No & No & Vaginal & No \\
\hline
\end{tabular}

EG: edad gestacional; CIA: comunicación interauricular; SPE: síndrome de preexcitación; PP: parto prematuro; NR: no reportado. *Promedio.

${ }^{\dagger}$ Sí/no o número de casos.

peso del neonato, en 11 hubo RCIU o bajo peso para la edad gestacional $(7,7 \%)^{10,16-18}$. Se produjeron $6(2,7 \%)$ muertes neonatales tempranas ${ }^{10,11,13,27,28}$, y $8(3,65 \%)$ neonatos tuvieron enfermedad cardiaca congénita ${ }^{28,29}$. En cuanto al desenlace de la preeclampsia, solo se documentó en tres casos ${ }^{10,12,30}$, incluida una de las que presentó accidente cerebrovascular ${ }^{12}$ (mencionado arriba), ninguno clasificado como grave y en gestantes de término. En la serie reportada por Lima et al, debido a la protección de la identidad de las pacientes, se reporta como $<10$ casos de $\mathrm{PE}^{17}$.

\section{Discusión}

La paciente que presentamos tenía CIA, el defecto anatómico más frecuentemente encontrado en la literatura, y si bien no presentó síndrome de preexcitación sí tenía evidencia de aumento del automatismo auricular que, por ser muy sintomático, requería el uso de medicación, en este caso un betabloqueador (metoprolol), el cual no se inició dado el RCIU grave (por la asociación poco clara entre los betabloqueadores y el impacto en el peso fetal ${ }^{31}$. Aunque el feto se encontraba por debajo de nuestro límite institucional de viabilidad, se respetó la decisión y el deseo de la paciente de continuar la gestación, en espera de un mejor desenlace neonatal. El aumento de cavidades derechas, la presencia de CIA y la elevación de catecolaminas durante la gestación predisponen a la presentación de $\operatorname{arritmias}^{32}$, que requieren manejo específico. Nuestra paciente cursó con PE de aparición temprana o precoz (PAP), que representa el $5-10 \%$ de todos los casos de $\mathrm{PE}$, y a nuestro entender es el primer caso reportado con esta asociación (AE y PAP), que se relaciona con 
la liberación a la circulación materna de factores antiangiogénicos (que son depresores miocárdicos). Tanto la CIA (flujo de izquierda a derecha) como posiblemente los factores antiangiogénicos pudieron favorecer la presentación del cuadro clínico de hipoxemia y disnea moderada. Si bien en la revisión de la literatura solo se documentaron tres casos de $\mathrm{PE}$ en pacientes con $\mathrm{AE}$ (en gestantes de término y no graves), los cambios hemodinámicos que ejerce por separado la gestación en AE y la PAP son un reto clínico. Por un lado, la PAP se presenta con un estado hipovolémico, de bajo gasto cardiaco y con una resistencia vascular periférica aumentada, con cambios del ventrículo izquierdo similares a una hipertrofia concéntrica, y puede explicar el llenado insuficiente del ventrículo izquierdo ${ }^{33-35}$. Y por otro lado, el aumento de la precarga en el ventrículo derecho, que es un cambio normal en la gestación, puede empeorar la regurgitación tricuspídea en las gestantes con $\mathrm{AE}$, por lo que el aumento de las cavidades derechas puede llevar a disfunción cardiaca izquierda ${ }^{13,16}$. El aumento del gradiente de presión por la regurgitación tricuspídea $>35 \mathrm{mmHg}$ durante la gestación se ha asociado a falla cardiaca ${ }^{16}$.

Se han documentado factores de riesgo de mal pronóstico en gestantes con cardiopatía, entre los que se encuentran la evaluación tardía por un equipo cardioobstétrico (posterior a las 20 semanas), la presencia de trastorno hipertensivo y el parto por cesárea ${ }^{36}$. En las pacientes con $\mathrm{AE}$, la presencia de cianosis, arritmia, CIA e insuficiencia tricuspídea grave con gradientes de presión elevados se asocia con desenlaces desfavorables, tal como ocurrió en la paciente que presentamos.

Considerando el escaso número de casos de pacientes con $\mathrm{AE}$ y $\mathrm{PE}$ reportados, no se puede establecer un nexo de causalidad, pero sí se debe alertar a los clínicos de esta asociación potencialmente mortal para la madre (si bien no se halló ninguna muerte materna en la revisión realizada), y que evidentemente se asocia a desenlaces neonatales desfavorables.

La evaluación antenatal (para control de la arritmia, reemplazo valvular y cierre de la CIA si están indicados), los controles prenatales y la atención del parto por un equipo cardioobstétrico en una institución con experiencia son fundamentales para la disminución de los eventos adversos, potencialmente letales ${ }^{37}$.

\section{Financiamiento}

La publicación es financiada con recursos propios.

\section{Conflicto de intereses}

Los autores declaramos que no tenemos ningún conflicto de intereses para la publicación del presente articulo.

\section{Responsabilidades éticas}

Protección de personas y animales. Los autores declaran que para esta investigación no se han realizado experimentos en seres humanos ni en animales.

Confidencialidad de los datos. Los autores declaran que han seguido los protocolos de su centro de trabajo sobre la publicación de datos de pacientes.

Derecho a la privacidad y consentimiento informado. Los autores han obtenido el consentimiento informado de los pacientes y/o sujetos referidos en el artículo. Este documento obra en poder del autor de correspondencia.

\section{Bibliografía}

1. Attenhofer Jost $\mathrm{CH}$, Connolly HM, Dearani JA, Edwards WD, Danielson GK. Ebstein's anomaly. Circulation. 2007;115:277-85

2. Baumgartner H, Falk V, Bax JJ, De Bonis M, Hamm C, Holm PJ, et al. 2017 ESC/EACTS Guidelines for the management of valvular heart disease. Rev Esp Cardiol. 2018;71:110.

3. Knight M, Bunch K, Tuffnell D, Shakespeare J, Kotnis R, Kenyon S, Kurinczuk, editores. MBRACCE-UK. Saving Lives, Improving Mothers' Care. Lessons learned to inform maternity care from the UK and Ireland Confidential Enquiries into Maternal Deaths and Morbidity 2015-17. Oxford; 2019. Disponible en: https://www.npeu.ox.ac.uk/assets/downloads/mbrrace-uk/reports/MBRRACE-UK\%20Maternal\%20Report\%20 2019\%20-\%20WEB\%20VERSION.pdf

4. Say L, Chou D, Gemmill A, Tunçalp O, Moller A-B, Daniels J, et al. Global causes of maternal death: a WHO systematic analysis. Lancet Glob Health. 2014;2:e323-33.

5. Roos-Hesselink J, Baris L, Johnson M, De Backer J, Otto C, Marelli A et al. Pregnancy outcomes in women with cardiovascular disease: evolving trends over 10 years in the ESC Registry Of Pregnancy And Cardiac disease (ROPAC). Eur Heart J. 2019;40:3848-55.

6. Regitz-Zagrosek V, Roos-Hesselink JW, Bauersachs J, Blomström-Lundqvist C, Cífková R, De Bonis M, et al. 2018 ESC Guidelines for the management of cardiovascular diseases during pregnancy. Eur Heart J. 2018;39:3165-241.

7. Silversides CK, Grewal J, Mason J, Sermer M, Kiess M, Rychel V, et al. Pregnancy outcomes in women with heart disease. J Am Coll Cardiol. 2018;71:2419-30

8. Misa VS, Pan PH. Evidence-based case report for analgesic and anesthetic management of a parturient with Ebstein's anomaly and Wolff-Parkinson-White syndrome. Int J Obstet Anesth. 2007;16:77-81.

9. Parizad R, Toufan Tabrizi M, Chenaghlou M. Maternal health and early outcome in pregnant woman with Eisenmenger syndrome and Ebstein anomaly. Int J Women Health Reprod Sci. 2014;2:35-8.

10. Donnelly JE, Brown JM, Radford DJ. Pregnancy outcome and Ebstein's anomaly. Heart. 1991;66:368-71.

11. Ranjbar A, Parizad R, Toufan Tabrizi M. Recurrent abortion in a patient with Ebstein anomaly. Int J Women Health Reprod Sci. 2014;2:265-7.

12. Zhao W, Liu H, Feng R, Lin J. Pregnancy outcomes in women with Ebstein's anomaly. Arch Gynecol Obstet. 2012;286:881-8.

13. Katsuragi S, Kamiya C, Yamanaka K, Neki R, Miyoshi T, Iwanaga N, et al. Risk factors for maternal and fetal outcome in pregnancy complicated by Ebstein anomaly. Am J Obstet Gynecol. 2013;209:452. e1-6.

14. Patel H, Jamnadas P. Undiagnosed Ebstein's anomaly in a pregnant woman. Rev Cardiovasc Med. 2014;15:276-80.

15. Rusdi L, Azizi S, Suwita C, Karina A, Nasution SA. Ebstein anomaly in pregnancy. Acta Med Indones. 2016;48:325-6. 
16. Kanoh $M$, Inai $K$, Shinohara $T$, Shimada $E$, Shimizu $M$, Tomimatsu $H$ et al. Influence of pregnancy on cardiac function and hemodynamics in women with Ebstein's anomaly. Acta Obstet Gynecol Scand. 2018:97:1025-31.

17. Lima FV, Koutrolou-Sotiropoulou P, Yen TYM, Stergiopoulos K. Clinical characteristics and outcomes in pregnant women with Ebstein anomaly at the time of delivery in the USA: 2003-2012. Arch Cardiovasc Dis. 2016;109:390-8.

18. Îñigo RCA, Torres GLG, Hernández HS, Gómez VJR. Anomalía de Ebstein y embarazo. Ginecol Obstet Mex. 2008;76:461-7.

19. Waickman LA, Skorton DJ, Varner MW, Ehmke DA, Goplerud CP. Ebstein's anomaly and pregnancy. Am J Cardiol. 1984;53:357-8.

20. Gouton M, Goldman C, Bakloul M, Sassolas F, Bozio A. Outcomes of pregnancy in women with Ebstein's anomaly. Eur Heart J. 2011;32 (Suppl. 1):23.

21. Castro FA, Díaz P, Calderón JC, Gutiérrez I. Cardiopatía y embarazo. Rev Colomb Anestesiol. 2011;39:207-14.

22. Sheikh AS, Lynch CM, Brockelsby J, Belham M, Gopalan F. Ebstein's anomaly $-a$ rare cause of shortness of breath in pregnancy. Eur $J$ Cardiovasc Med. 2012;2(1).

23. Groves ER, Groves JB. Epidural analgesia for labour in a patient with Ebstein's anomaly. Can J Anaesth. 1995;42:77-9.

24. Lesniak-Sobelga AM, Kostkiewicz M, Wisniowska-Smialek S, Biernacka B. Pregnancy in patients with Ebsteins anomaly - echocardiographic and clinical study. Eur Heart J Cardiovasc. 2015;5 (Suppl 2):ii34.

25. Littler WA. Successful pregnancy in a patient with Ebstein's anomaly. Heart. 1970;32:711-3.

26. Parizad R, Toufan Tabrizi M, Chenaghlou M. Maternal health and early outcome in pregnant woman with Eisenmenger syndrome and Ebstein anomaly. Int J Women's Health Reprod Sci. 2014;2:35-8.
27. Katamreddy A. Ebstein's anomaly in a tachycardic postpartum woman J Am Coll Cardiol. 2019;73(9 Suppl 1):2518.

28. Chopra S, Suri V, Aggarwal N, Rohilla M, Vijayvergiya R, Keepanasseril A. Ebstein's anomaly in pregnancy: maternal and neonatal outcomes. J Obstet Gynaecol Res. 2010;36:278-83.

29. Connolly HM, Warnes CA. Ebstein's anomaly: outcome of pregnancy. J Am Coll Cardiol. 1994;23:1194-8.

30. Houser L, Zaragoza-Macias E, Jones TK, Aboulhosn J. Transcatheter closure of atrial septal communication during pregnancy in women with Ebstein's anomaly of the tricuspid valve and cyanosis. Catheter Cardiovasc Interv. 2015;85:842-6.

31. Duan L, Ng A, Chen W, Spencer HT, Lee M-S. Beta-blocker subtypes and risk of low birth weight in newborns. J Clin Hypertens (Greenwich). 2018;20:1603-9

32. Muñoz Ortiz E, Agudelo JF, Velásquez J, Arévalo EF. Arritmias en la paciente embarazada. Rev Colomb Cardiol. 2017;24:388-93.

33. Nelson DB, Ziadie MS, Mclntire DD, Rogers BB, Leveno KJ. Placental pathology suggesting that preeclampsia is more than one disease. Am J Obstet Gynecol. 2014;210:66.e1-7.

34. Valensise H, Vasapollo B, Gagliardi G, Novelli GP. Early and late preeclampsia. Hypertension. 2008;52:873-80.

35. Valensise H, Vasapollo B, Gagliardi G, Novelli GP. Early and late preeclampsia: two different maternal hemodynamic states in the latent phase of the disease. Hypertension (Dallas, Tex 1979). 2008;52:873-80.

36. Muñoz-Ortiz E, Gándara-Ricardo JA, Velásquez-Penagos JA, Giraldo-Ardila N, Betancur-Pizarro AM, Arévalo-Guerrero EF, et al. Factores de riesgo para complicaciones cardíacas, obstétricas y neonatales en pacientes con enfermedad cardíaca durante el embarazo. Arch Cardiol Mex. 2020;90:115-21.

37. Mehta LS, Warnes CA, Bradley E, Burton T, Economy K, Mehran R, et al. Cardiovascular considerations in caring for pregnant patients: a scientific statement from the American Heart Association. Circulation. 2020;141:e884-903. 